\title{
Long-range geometrical correlations in two-dimensional foams
}

\author{
B Dubertret $\dagger$, N Rivier $\dagger$ and M A Peshkin $\ddagger$ \\ $\dagger$ Laboratoire de Dynamique des Fluides Complexes, Université Louis Pasteur, 3 rue de \\ l'Université, Strasbourg 67084, France \\ $\ddagger$ Mechanical Engineering Department, 2145 Sheridan Road, Northwestern University, Evanston, \\ IL 60208-3111, USA
}

Received 6 May 1997

\begin{abstract}
The statistical properties of two-dimensional, space-filling random cellular structures (foams, or their dual, random triangulations) in statistical equilibrium are obtained by maximum entropy inference and topological simulations. We show by maximum entropy inference that for a broad class of foams (shell-structured, including three-sided cell inclusions), all two-cell topological correlators $A_{j}(k, n)$ (average number of pairs of $k$-cell and $n$-cell at a topological distance $j$ ) are linear in $n$ and $k$, the numbers of neighbours of the cells. This generalizes a correlation known for neighbouring cells $(j=1)$ which implies the linearity of Aboav's relation (between the total number of neighbours of the cells adjacent to a $n$-neighboured cell and $n$ ). Our results, verified by simulations, also build up Gauss's theorem for cellular structures. Any additional restriction in exploring local cell configurations, besides the constraints of filling space at random, will manifest itself through a deviation from linearity of the correlators $A_{j}(k, n)$ and the Aboav relation. Notably, foams made of Feynman diagrams have additional, contextdependent restrictions and their Aboav relation is slightly curved. It is essential that the local random variable $n$ denotes the number of neighbours of the cell and not that of its sides, whenever the two are different.
\end{abstract}

\section{Introduction}

Many natural materials may be depicted, at a structural level, as a random filling of space by cells, polyhedra in three dimensions (3D) (biological tissues (Thompson 1942), metallurgical aggregates (Aboav 1970)) or polygons in two-dimensions (2D) (honeycomb, butterfly wings). In $2 \mathrm{D}$, the random cellular foam is a planar, regular graph of valency three.

Most of the structural studies have been carried out on two-dimensional trivalent cellular systems (2D foams or cuts of a 3D foams), very common in nature (Weaire and Rivier 1984, Gibson and Ashby 1988). Their characterization includes in general the distributions of metric and topological properties of single cells (number of sides, area, edges length, cell perimeter). In addition to the geometrical correlations discussed in this paper, the main statistical properties are summarized by Lewis's (1928) law which links the area of a cell to the number of its sides, by von Neumann's law, describing the growth rate of cells, and finally by scaling laws (Mullins 1956, Stavans and Glazier 1989)). All characterize the steady-state evolution of the foam (statistical equilibrium under local topological transformations).

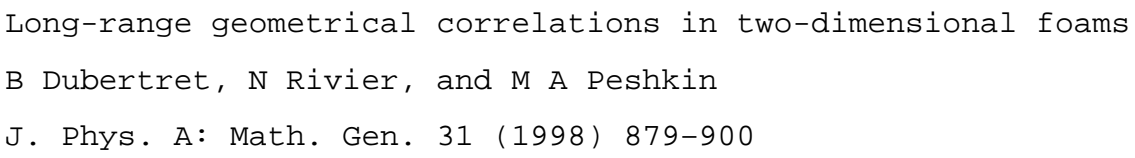


For reasons that will become clearer in the following sections, we have distinguished two types of foams.

- Foams for which each $k$-sided cell has $k$ different neighbours. We name them natural foams since it is always the case in natural structures. In such structures, no cell is its own neighbour and no neighbours share more than one side. In the terminology of Feynman diagrams, natural foams have no tadpoles or self-energy parts.

- Foams where some $k$-sided cells have less than $k$ neighbours, as is the case when an edge is 'decorated' by two neighbouring three-cells. In such foams, the relevant topological property is the number of neighbours of a cell, not the number of its sides. A $k$-cell is then a cell with $k$ neighbours ( $k$-neighboured) and $P_{k}$ is the probability that a $k$-neighboured cell exists. Such foams appear in topologically simulated systems. We name them artificial foams.

Up until now, only natural foams (for which the numbers of neighbours and sides are equivalent) have been studied.

Natural foams can be generated by choosing topological moves that keep all cells convex. A simple example of such dynamics is cells fragmentation (Delannay and Le Caër 1994). Alternatively, natural foams can be produced artificially by rejecting explicitly the last move (neighbour switch) that would produce a forbidden configuration (cell sharing more than one side with one of its neighbours or being its own neighbours). This is an additional restriction (containing specific information) imposed on the system exploring configuration space.

Two neighbouring cells have their topological properties correlated. In natural foams, this correlation is given by a semi-empirical law, the Aboav-Weaire's law (Aboav 1970, Weaire 1974). It states that, on average, $n \cdot m(n)$, the total number of sides of the cells adjacent to any $n$-sided cell ( $n$-cell) is linearly related to $n$ by:

$$
n \cdot m(n)=(6-a) n+6 a+\mu_{2}
$$

where $\mu_{2}$ is the second moment of the distribution $\left(P_{n}\right)$ of the number $n$ of edges of cells: $\mu_{2}=\left\langle n^{2}\right\rangle-\langle n\rangle^{2}$. In 2D, $\langle n\rangle=6$ is a mathematical law (a consequence of Euler's relation on an infinite, planar trivalent graph). Relation (1) depends on a single parameter $a$ (as a consequence of the sum rule: $\langle n \cdot m(n)\rangle=\mu_{2}+36$ (Weaire 1974)), which is positive and of the order of 1 in natural structures (Rivier 1993b).

In general, one can define the two-cell characteristics $M_{k}(n)$ (the average number of $k$ cells neighbouring an $n$-cell), or the correlators $A_{1}(k, n)\left(A_{1}(k, n)=M_{k}(n) / P_{k}=A_{1}(n, k)\right.$ is the average number of pairs of neighbouring $k$-cell and $n$-cell, given that the two cells exist). The quantity $n \cdot m(n)$, defined for natural foams only, can be related to $M_{k}(n)$ or the correlators $A_{1}(k, n)$ as:

$$
n \cdot m(n)=\sum_{k} k M_{k}(n)=\sum_{k} k P_{k} A_{1}(k, n) .
$$

The correlator $A_{1}(k, n)$ is a strictly positive, symmetric function of $k$ and $n$ independent of the probability distribution $P_{k}$. It is predicted to vary linearly with $k$ and $n$, by arguments of maximum entropy first introduced in cellular networks in Rivier and Lissowski (1982), and further developed in Peshkin et al (1991) (see also section 2). It is found that in the case of natural foams (Le Caër and Delannay 1993):

$$
A_{1}(k, n)=\sigma(k-6)(n-6)+n+k-6
$$

$\sigma=-a / \mu_{2}$. In order to have larger cells neighbours of smaller cells and vice versa, $\sigma$ is restricted to: $\frac{1}{6} \geqslant \sigma$, as discussed in Rivier (1993a). 
The theories that have been used hitherto to characterize the evolution of 2D foams have all neglected correlations beyond first neighbours, assuming therefore that longer-ranged correlations are negligible for the foam in a stationary state (scaling state, characterized by a stationarity distribution $P_{k}$ ). However, recent studies (Aste et al 1996b) tend to indicate that correlations beyond nearest neighbours may not be negligible in soap froths.

\subsection{Topological distance and shell-structured inflatable foams}

At this stage, it is useful to define a topological distance between two cells (Aste et al 1996a). The distance $j$ between two cells A and B is the smallest number of edges crossed by paths connecting A and B. Any cell A can be taken as the 'germ' cell, $j=0$. Then, all 2D foam, natural or artificial can be foliated into concentric layers of equidistant $(j=1,2, \ldots)$ cells. In between successive layers, there may be cells or clusters of cells which are localized defect inclusions. A foam without defect inclusions is called shell-structured inflatable (SSI) (Aste et al 1996a).

The layer of cells at distance $j$ encloses layers $j-1, j-2, \ldots, 0$ (the germ cell). It includes all the cells at distance $j$ which are themselves neighbours of at least one cell at distance $j+1$. The cells that do not obey this condition make up the defect inclusions (between layers $j-1$ and $j$ ). Three-sided cells (other than germ cells) are defect inclusions. So are tadpoles and edge renormalizations. Thus, an SSI foam is necessarily a natural foam. But there are natural foams that are not SSI (for examples those with three-sided cells). Examples of defect inclusions are given in figure 1. Disorder makes the concentric layers wiggly. Defects lie in between the folds. Asymptotically, as $j \rightarrow \infty$, the number of cells in layer $j$ increases linearly with the distance $j$, independently of the number of neighbours $n$ of the germ cell, as space-filling and foliation require. This behaviour, confirmed in simulations (Ohlenbusch et al 1997), imposes weak conditions on the hitherto arbitrary parameters $\sigma_{j}$ (see equations (22) and (23)). Obviously, the average size of each localized defect inclusion is independent of $j$ at large distances $j$.

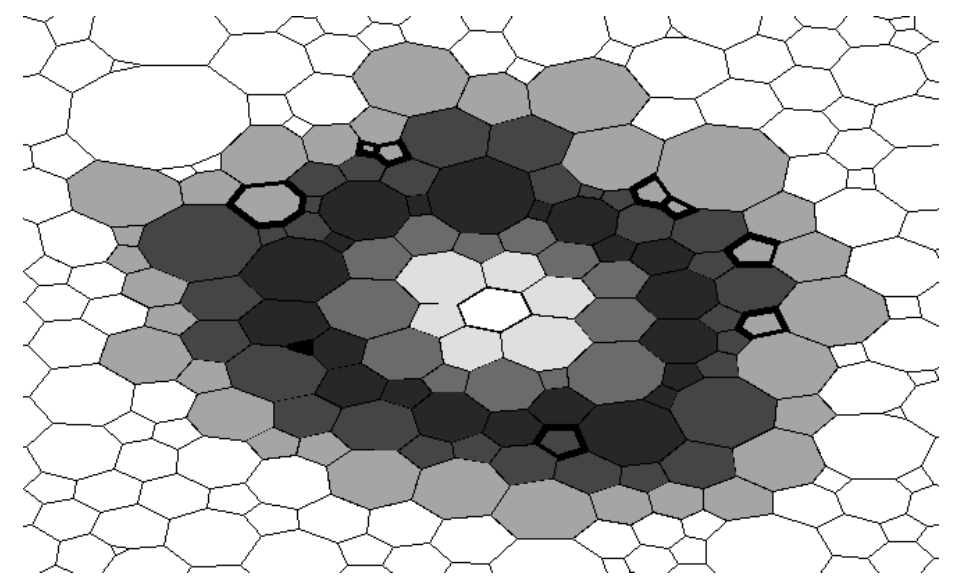

Figure 1. An example of the shell structure around a germ cell (in white). Layers 1-5 are represented in different types of grey. The defects inclusions are outlined with a heavy curve. 


\subsection{Outline}

The purpose of this paper is to investigate correlations beyond nearest neighbours. We obtain their analytical form for a broad class of foams (SSI (Aste et al 1996a) which may include three-sided cell inclusions) by generalizing the maximum entropy arguments that inferred the linearity of $A_{1}(k, n)$. We then compare these analytical results with results of topological simulations (which deal with artificial foams). SSI foams are included in the class of natural foams, which belong to the class of artificial foams. This paper is divided into seven sections.

In section 2 we discuss the latest results obtained on the Aboav law. Godrèche et al (1992) gave an analytical expression for the Aboav's law which is in apparent contradiction with the linearity predicted from maximum entropy arguments. We show that there is no contradiction: the deviation from linearity in Godrèche et al is due to additional contextdependent constraints specific to their representation of the foam (bosonic $\phi^{3}$ Feynman diagrams). Indeed, we show that provided the dynamics of the system is suitably chosen, the linearity of $A_{1}(k, n)$ in $k$ and $n$ can be inferred from maximum entropy arguments for any kind of foams, artificial or natural.

In section 3 we generalize the first neighbour's correlator $A_{1}(k, n)$ to cells $j$ layers apart with the correlator $A_{j+1}(k, n)$. To do so, we use the layer representation of foams developed in (Aste et al 1996a).

In section 4 we focus on $A_{2}(k, n)$. We prove its linear dependence in $k$ and $n$. For didactic reasons we discuss first the case of SSI foams. A more general case including three-cells will be presented.

These results are extended to $A_{j}(k, n)$ for arbitrary $j$ in section 5. An explicit analytic expression for $A_{j}(k, n)$ is given for all $j$, for SSI foams with three-cells inclusions.

Section 6 infers the linearity of $A_{j}(k, n)$ for foams where $P_{3} \neq 0$, under some restriction for $A_{j}(3, n)$.

These analytic results are compared with simulations in section 7 .

Hereafter, a $k$-cell denotes a $k$-neighboured cell (which in the case of natural foams is also a $k$-sided cell), and $P_{k}$ is the probability of finding a $k$-neighboured cell.

\section{Discussion of the linearity of Aboav's relation}

Aboav (1970) first noted a correlation between the number of sides $n$ of a cell and the average total number of sides $n \cdot m(n)$ of its $n$ neighbours. In all known natural and man-made structures, it is found empirically that $n \cdot m(n)$ is linearly related to $n$.

This linear form was first explained through maximum entropy arguments by Peshkin et al (1991) for a system subject to a minimal number of topological constraints. A year later, Godrèche et al (1992) gave an analytical expression for the topological correlators $A_{1}(k, n)$ and the Aboav's law. They found, for a natural foam constructed from the Feynman's diagrams of a $\phi^{3}$ fields theory, $n \cdot m(n)=7 n+3+9 /(n+1)$. In both case, simulations were in very good agreement with the results obtained. However, the slopes were different (7.33 in Peshkin et al and 7 in Godrèche et al) and the deviations from linearity $9 /(n+1)$ in Godrèche et al perceptible in the simulations as an upturn at small $n$ was not explained by maximum entropy inference.

In this section we propose to resolve these apparent contradictions.

Let us summarize the maximum entropy argument in the case of artificial foams.

The topology of cellular systems imposes constraints on the local configuration of the cell assembly, i.e. on the probability distribution $P_{n}$ in 2D. The number of neighbours $n$ of 
a cell is the only local random variable of the problem. Other incidence and coordination numbers are fixed. For example, vertices are always trivalent.

The constraints are:

$$
\begin{aligned}
& \sum_{k} P_{k}=1 \\
& \sum_{k} k P_{k}=\alpha \\
& \sum_{k} P_{k} A_{1}(n, k)=n .
\end{aligned}
$$

This is the minimal number of constraints (averaged over the stationary distribution $P_{k}$ ) that a topological system has when only first neighbours correlations are taken into account.

$\alpha$ is exactly 6 in the case of natural foams and is a given constant less than 6 in the case of artificial foams. Note that the average number of sides of a cell is always 6 for both natural and artificial foams. It is a consequence of the Euler relation, which depends solely on the fact that in any foam, vertices are the meeting point of three and only three edges.

Since every $k$-neighboured cell has $k$ neighbours (it is a tautology), the set of constraints (6) does not contain any irreducible information that is not already in (4) or (5). In such case, maximum entropy infers that, as the system reaches statistical equilibrium, each constraint of the set (6) becomes redundant with the first two. This is the case if $A_{1}(k, n)$ is linear in $k$ and $n$. The total number of constraints is thus reduced and the number of different local realizations of the foam with a distribution $P_{k}$ (i.e. the entropy of the system) are increased. This general argument is the basics of statistical physics. It requires the system to be allowed to explore the space of local configurations (phase space) without any restriction, apart from those imposed by (4)-(6). This exploration is performed by local elementary topological transformations which shuffle the local random variable $k$ (the equivalent in thermodynamics of collisions between gas molecules or between the molecules and walls).

Had we considered the number of sides of a cell instead of its number of neighbours, the set of constraints (6) would have contained additional irreducible information. The smallest value of $n$ for which the number of sides and the number of neighbours may differ is $n=6$ (decoration of one edge of a three-cell by a pair of neighbouring three-cells). Thus, if one writes $\sum_{k} P_{k} A_{1}(6, k)=6$ with $k$ and $n=6$ representing the numbers of sides, this implies that the decoration of one edge of a three-sided cell has been explicitly forbidden. If this condition is not naturally respected by the way the system evolves, it is independent, additional information. Consequently, $\sum_{k} P_{k} A_{1}(6, k)=6$ cannot be made into a linear combination of other constraints. The same reasoning is true for $n$ greater than 6. Obviously, when the dynamics keep the foam natural, there is no difference in the number of sides and of neighbours of a cell and no restriction in the exploration of the space of the configuration. Maximum entropy inference applies, and yields linearity of $A_{1}(k, n)$, as observed in the simulations (Delannay and Le Caër 1994).

The Feynman diagrams calculated by Tutte (1962) and used by Godrèche et al excluded tadpoles and self-energy inclusions, thereby excluding from the foam a cell which is its own neighbour and two cells which are separated by more than one edge. To achieve this in their simulation they did not permit the flip leading to a tadpole or self-energy inclusion. The addressed edge is left unflipped. This restriction is context-dependent. The initial state, the final state and the move are not enough to decide whether the move is accepted. This is a situation that cannot be depicted by maximum entropy arguments (see the appendix). Note that this discussion does not invalidate the enumeration and simulation made in Godrèche 


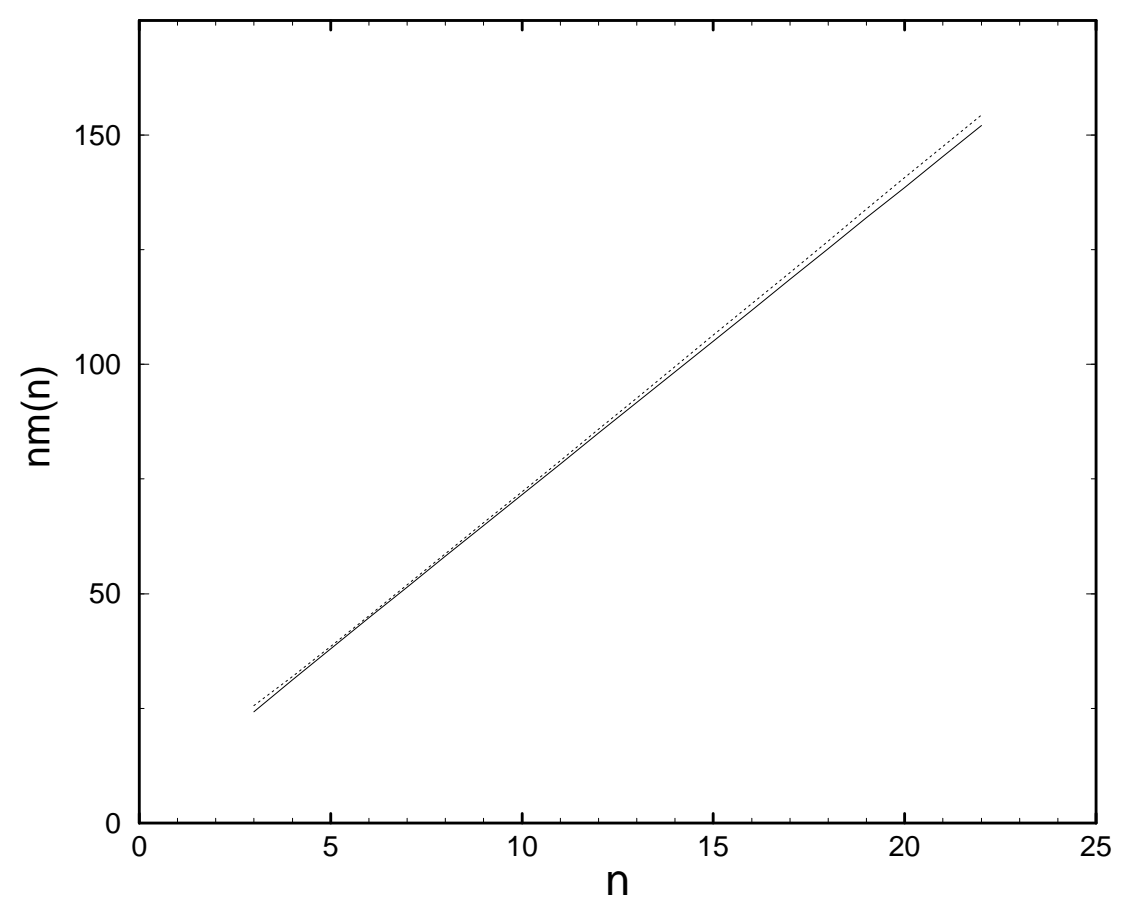

Figure 2. Aboav's relation obtained for a foam without restriction (full line) and with the restrictions imposed by Godrèche (dotted line).

et al which are exact.

In figure 2, we compare Aboav's relation obtained in the simulation with the additional restriction of Godrèche et al with the one obtained without additional restriction on the system (i.e. we permit two neighbouring cells to share more than one side). Neighbours instead of sides are labelled and counted. This precaution is irrelevant for natural foams, but is essential for the (highly) artificial foam produced in the second case.

In figures 3 and 4 , we plot the topological correlators $A_{1}(k, n)$ for both foams. As expected, the $A_{1}(k, n)$ of the unrestricted simulation are linear in $k$ and $n$.

Unless otherwise stated, all simulations in this paper are made with the method used in Peshkin et al ( $D=0$, neighbours exchange (T1) only, edges addressed at random).

In sections 3-5, we consider a system that reached statistical equilibrium through dynamics that produces a natural foam. The notion of $k$-neighboured cells and that of $k$-sided cells are thus equivalent.

\section{Correlations beyond the first neighbours}

The system of constraints (4)-(6) is minimal but not comprehensive. In particular, there are topological correlations between two cells that are not direct neighbours, i.e. without an edge in common. To study these correlations, we generalize the correlator used for the nearest neighbours.

As first suggested by Aste (private communication, see also Szeto et al (1997)), we define $P_{k} A_{j}(k, n)$ as the average number of $k$-cells at a distance $j$ from a $n$-neighboured germ cell. Thus, $P_{k} A_{1}(k, n)$ (discussed in the previous section) is the average number of 


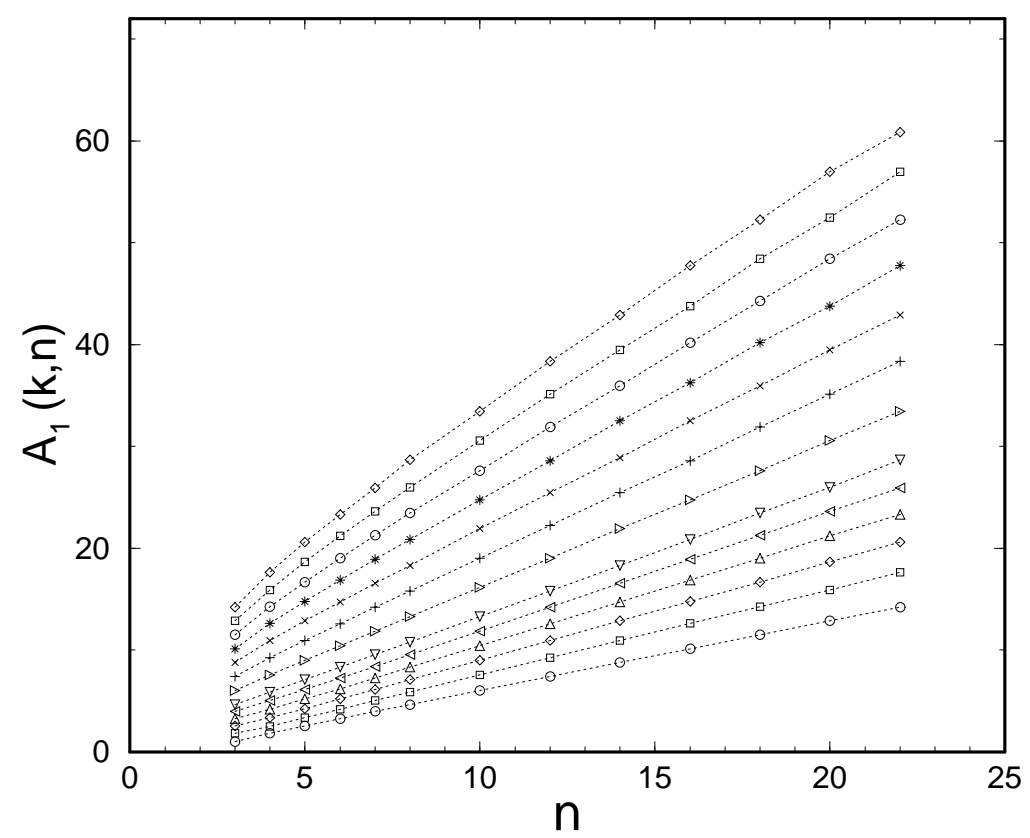

Figure 3. Topological correlators for a foam without restriction. For $k>8$, only the $A_{1}(k, n)$ for $k$ even are plotted. Bottom line, $k=3$. Top line, $k=22$.

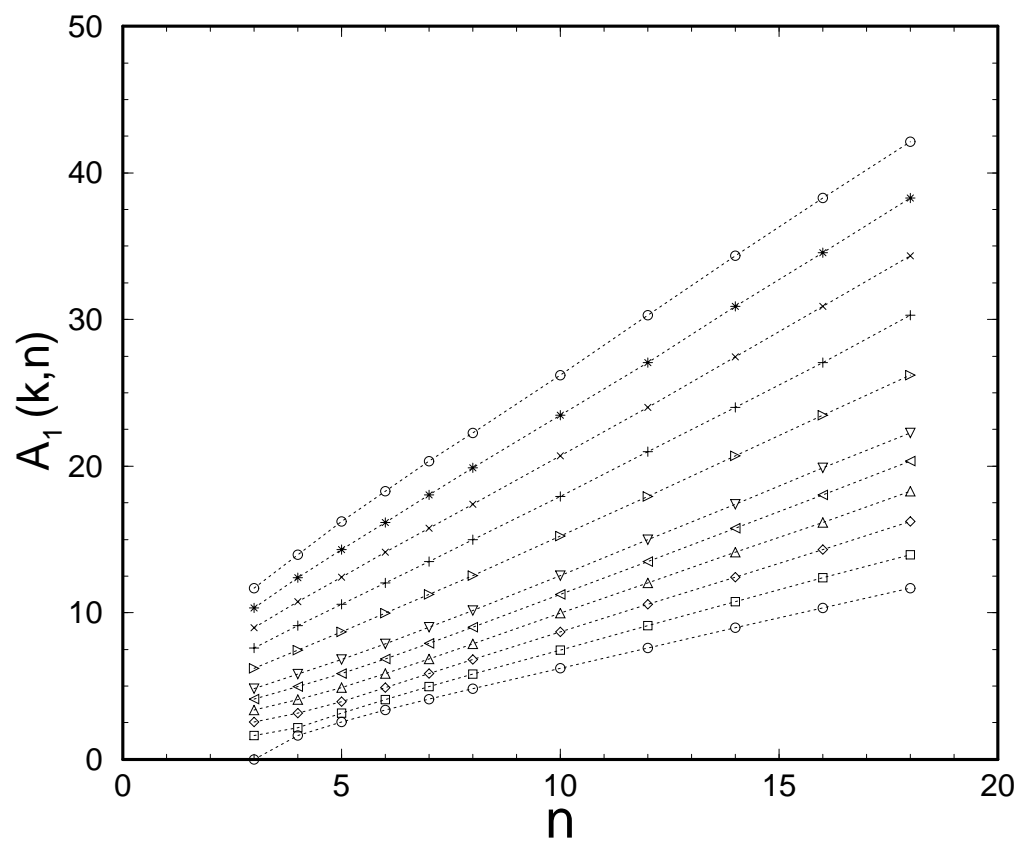

Figure 4. Topological correlator for a (natural) foam with the restrictions imposed by Godrèche. For $k>8$, only the $A_{1}(k, n)$ for $k$ even are plotted. 
$k$-cells, nearest neighbours to the $n$ germ-cell. $P_{k}$ is the probability that a $k$-cell exists. It is defined as the ratio of the number $N(k)$ of $k$-cells to the total number of cells in the system $N_{T}$.

$P_{k} A_{j}(k, n)$ is equal to the ratio of the number of couples $C^{j}(n, k)$ of $n$-cell and $k$-cell separated by $j-1$ layers (each couple is counted once) to the number $N(n)$ of $n$-cells in the system: $P_{k} A_{j}(k, n)=C^{j}(n, k) / N(n)$.

This defines the symmetric quantity

$$
A_{j}(k, n)=C^{j}(n, k) N_{T} / N(n) N(k)=A_{j}(n, k)
$$

which measures the topological correlations in foams as a function of the layer distance $j$. It may be viewed as the average number of pairs of $k$-cell and $n$-cell, $j$ layers apart, given that the two cells exist. The functional dependence of this correlator will be the subject of the rest of the paper.

It is useful to introduce $K_{j}(n)$, the mean number of cells at a distance $j$ from a given $n$-cell. It is equal to the average of $A_{j}(k, n)$ over $P_{k}$, the distribution of $k$-cells for $n$ fixed:

$$
\sum_{k} P_{k} A_{j}(k, n)=K_{j}(n) .
$$

In the case $j=1$, i.e. in the case of nearest neighbours, $K_{1}(n)=n$ and equation (8) is the constraint (6). When $j>1$, (8) is a new constraint on the system.

When $j=2, K_{2}(n)$ is the average number of second neighbours of a $n$-cell. As already noted in Le Caër and Delannay (1993), in the case of natural foams, it is simply related to the Aboav's law: $K_{2}(n)=n \cdot m(n)-4 n-P_{3} A_{1}(3, n)$. This shows that when statistical equilibrium is reached, $K_{2}(n)$ is itself a linear function of $n$, since $A_{1}(k, n)$ and $n \cdot m(n)$ are linear in $n$ as established in section 2 .

The consequences of this linearity are discussed in the next section.

\section{Second neighbour correlations for SSI foams}

\subsection{Sum rules}

In this section, we only consider SSI foams, which, incidently, have no three-sided cells. Two main reasons motivate our choice. First, in natural 2D foams there are very few threecells (soap froth, metallurgical aggregates) or none at all (epidermis (Lewis 1928, Dubertret and Rivier 1997). Other non-three-sided defects are rare in natural foams (Aste et al 1996b). The second reason is simplicity: the iteration of correlations and constraints hold strictly only for SSI foams. In the next section we will see how three-cells can be included in the discussion. They are local inclusions-or vertex decorations-in the foliation of the foam into layers that can be eliminated by a $\mathrm{T} 2$ transformation affecting only the nearest neighbours.

To begin with, we note that there are two types of second neighbours. Those linked to the germ cell with one arm (arm second neighbours) and those without direct edge-link with the germ cell (free second neighbours). Free does not imply that there are no correlations between the two cells. Because of this difference in physical linkage, arm second neighbours and free second neighbours are expected to have different correlations with the germ cell.

On average, the total number of second neighbours of a given $n$-cell, $K_{2}(n)$ may be written in two different ways.

- Directly with the second-neighbour correlator $A_{2}(k, n)$ (equation (8) for $j=2$ ):

$$
\sum_{k} P_{k} A_{2}(k, n)=K_{2}(n) \text {. }
$$


- By using the first-neighbour correlator $A_{1}(k, n)$

$$
\sum_{k \geqslant 4}(k-4) P_{k} A_{1}(k, n)=K_{2}(n) .
$$

Out of the $k$ edges of each first neighbour, only $k-4$ edges separate it from second neighbours (each second neighbour is counted once).

Similarly, the average number of free second neighbours $K_{2}^{\text {free }}(n)$ of a given $n$-germ cell may be computed using the $A_{1}(k, n)$ :

$$
\sum_{k \geqslant 5}(k-5) P_{k} A_{1}(k, n)=K_{2}^{\mathrm{free}}(n) .
$$

This, in turn defines $A_{2}(k, n)^{\text {free }}$ (the same physical quantity as $A_{2}(k, n)$ but pertaining only to the free second neighbours) as

$$
\sum_{k} P_{k} A_{2}(k, n)^{\mathrm{free}}=K_{2}^{\mathrm{free}}(n) .
$$

The difference between equations (10) and (11) gives the average number of arm second neighbours of a $n$ germ cell, $K_{2}^{\mathrm{arm}}(n)$ :

$$
\sum_{k \geqslant 4} P_{k} A_{2}^{\mathrm{arm}}(k, n)=n-P_{4} A_{1}(4, n)=K_{2}^{\mathrm{arm}}(n)
$$

written with $A_{2}(k, n)^{\text {arm }}$ (the same physical quantity as $A_{2}(k, n)$ but only germane to the arm second neighbours). Note that a given $n$ cell has $n$ arms, but the number of its arm second neighbours may be inferior to $n$, if one or more of its nearest neighbours have four sides. The sums over $k \geqslant 4$ are, here, unrestricted.

\subsection{Functional form of $A_{2}(k, n)$}

Up to second neighbours, the distribution $P_{k}$ obeys the following sum rules:

$$
\begin{aligned}
& \sum_{k} P_{k}=1 \\
& \sum_{k} k P_{k}=6 \\
& \sum_{k} P_{k} A_{1}(k, n)=K_{1}(n) \\
& \sum_{k} P_{k} A_{2}(k, n)=K_{2}(n) .
\end{aligned}
$$

The third sum rule is redundant with the first two and $A_{1}(k, n)$ is linear in $n$ as seen in section 2. It then follows from equation (10) that $K_{2}(n)$ is linear in $n$. The last sum rule is therefore a constraint on $P_{k}$, which can in turn be made redundant if $A_{2}(k, n)$ is linear in $k$ and in $n$. The entropy is therefore maximized.

With equations (14) and (10), and using the linear form of $A_{1}(k, n)$ for natural foams given in the introduction, we can compute the analytical form of $A_{2}(k, n)$ :

$$
A_{2}(k, n)=(k-6) \sigma_{2}(n-6)+\left(\mu_{2} \sigma_{1}+2\right)(n+k-6)+\mu_{2}\left(1-6 \sigma_{1}\right)
$$

where $\sigma_{1}$ stands for the $\sigma$ of equation (3) and $\sigma_{2}$ is a new parameter. 


\subsection{Functional form of $A_{j}(k, n)$}

This is the first step of an iterative process which uses the linearity of $A_{j}(k, n)$ to infer the linearity of $K_{j+1}(n)$ and, by maximum entropy arguments, that of $A_{j+1}(k, n)$. Equation (10) for $j>1$ reads in general:

$$
\sum_{k}(k-4) P_{k} A_{j}(k, n)=K_{j+1}(n)+K_{j-1}(n)
$$

where $K_{0}(n)=0, K_{1}(n)=n$ and $A_{1}(k, n)$ are linear in $n$.

The iterative process is set up as follows. If $A_{j}(k, n)$ are linear in $n, K_{j+1}(n)$ is linear in $n$ by (16). Equation (8):

$$
\sum_{k} P_{k} A_{j+1}(k, n)=K_{j+1}(n)
$$

becomes a constraint and can be made redundant with (4) and (5) if $A_{j+1}(k, n)$ is linear in $n$, thereby maximizing the entropy of the system.

The most general functional form of $A_{j+1}(k, n)$ is then given by:

$$
A_{j+1}(k, n)=(k-6) \sigma_{j+1}(n-6)+\alpha_{j+1}(n+k-6)+\beta_{j+1}
$$

where $\sigma_{j+1}$ is a new parameter and $\alpha_{j+1}$ and $\beta_{j+1}$ can be computed iteratively as functions of the $\sigma_{j}$, by using (16) and (17). The first values of $\alpha$ and $\beta$ are: $\alpha_{0}=0, \beta_{0}=0, \alpha_{1}=1$, $\beta_{1}=0$.

Relation (18) implies that $K_{j+1}(n)=n \alpha_{j+1}+\beta_{j+1}$. The sum rule (16) may then be written:

$$
\sum_{k}(k-4) P_{k} A_{j}(k, n)=n\left(\alpha_{j+1}+\alpha_{j-1}\right)+\beta_{j+1}+\beta_{j-1}
$$

with:

$$
\begin{aligned}
& \alpha_{j+1}=-\alpha_{j-1}+2 \alpha_{j}+\mu_{2} \sigma_{j} \\
& \beta_{j+1}=-\beta_{j-1}+2 \beta_{j}+\mu_{2}\left(-6 \sigma_{j}+\alpha_{j}\right) .
\end{aligned}
$$

The solutions of this system are:

$$
\begin{aligned}
\alpha_{j+1} & =\mu_{2} \sum_{k=1}^{j}(j-k+1) \sigma_{k}+j+1 \\
\beta_{j+1} & =\mu_{2} \sum_{k=1}^{j}(j-k+1)\left(\alpha_{k}-6 \sigma_{k}\right) \\
& =\mu_{2}^{2} \sum_{k=1}^{j}\left(\begin{array}{c}
j-k+2 \\
3
\end{array}\right) \sigma_{k}+\mu_{2}\left(\begin{array}{c}
j+2 \\
3
\end{array}\right)-6 \mu_{2} \sum_{k=1}^{j}(j-k+1) \sigma_{k} .
\end{aligned}
$$

Asymptotically, one expects $\alpha_{j}(n)$ to be independent of the germ cell, i.e. of $n$. This symmetry is a local gauge invariance (Rivier et al 1998). Thus, as $j \rightarrow \infty, \beta_{j}$ is linear in $j$, as observed (Ohlenbusch et al 1997).

\section{Correlations in the presence of three-sided cells}

Let us see how the presence of three-cells alters the results obtained so far.

A natural foam with three-cells can be obtained from a natural foam without three-cells by decorating some vertices into three-cells as shown in figure 5. A three-cell in layer $j$ results from the decoration of a vertex on the closed contour separating layers $(j-1)$ 


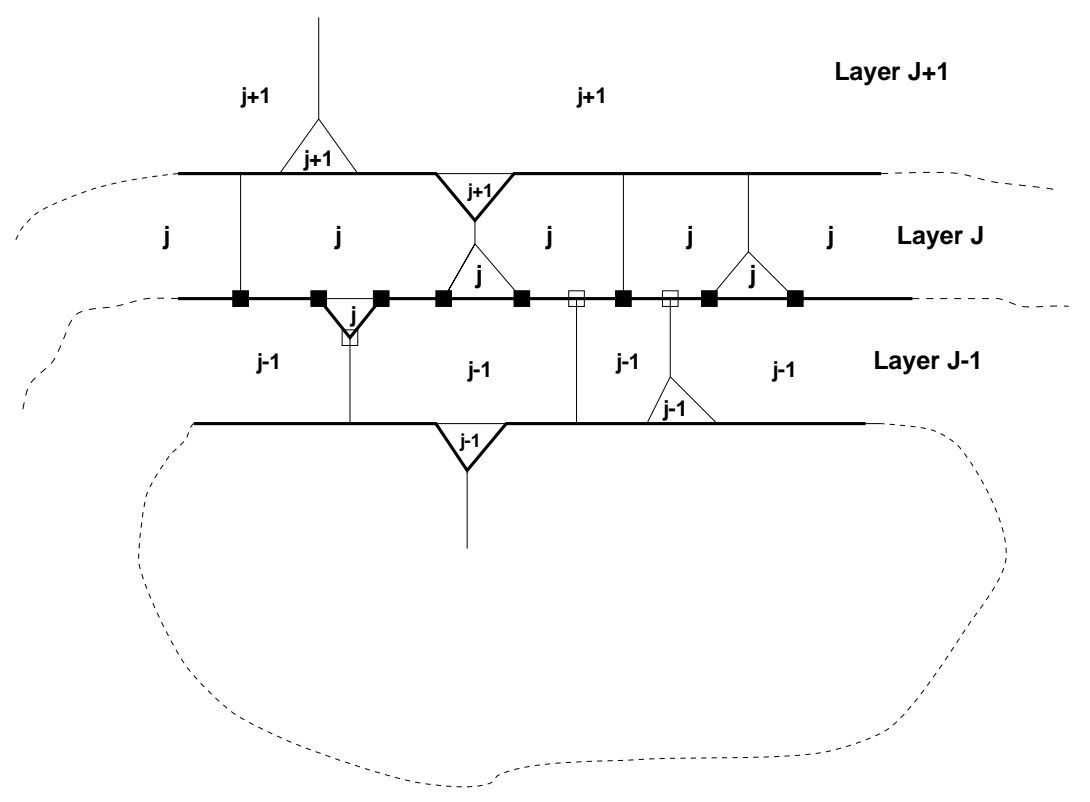

Figure 5. Schematic representation of a part of layer $j$. Cells are labelled by the layer to which they belong. Heavy lines separate the different layers, full squares (resp. open squares) are the vertices pointing up $V_{j}^{\text {up }}(n)$ (resp. down $\left.V_{j}^{\text {down }}(n)\right)$ on the line separating layer $(j-1)$ and $j$. The central cell (layer $j=0$ ) has $n$ neighbours.

and $j$. It is always a defect. There are on average $P_{3} A_{j}^{\text {up }}(3, n)$ three-cells pointing up and $P_{3} A_{j}^{\text {down }}(3, n)$ three-cells pointing down.

With these definitions, equation (16), obtained for a SSI foam, can be generalized to include three-sided cells defects as:

$$
\begin{gathered}
\sum_{k \geqslant 4}(k-4) P_{k} A_{j}(k, n)=K_{j+1}(n)+K_{j-1}(n)+P_{3}\left(A_{j+1}^{\text {down }}(3, n)\right. \\
\left.+A_{j}^{\text {down }}(3, n)+2 A_{j}^{\text {up }}(3, n)-A_{j-1}(3, n)\right) .
\end{gathered}
$$

$K_{j}(n)$ numbers all cells at distance $j$ from the germ cell, including now three-cells.

Since there is no general relation for $A_{j+1}(3, n)$ in (24) and (25) below, we assume that the $A_{j}^{\text {down }}(3, n)$ are linear in $n$.

This assumption (supported by the simulations in section 6) can be justified by the construction of the foam. The number of three-cells down or up in layer $j$ is proportional to the number of cells (linearly dependent on $n$ by (17)) before any three-cell were introduced.

The iteration procedure of the previous section becomes then possible. It infers the linearity of $A_{j}(k, n)$ for a SSI foam containing three-sided cells as defects.

\subsection{Topological Gauss theorem}

Equation (24) can be written, summing over all cells, as:

$$
\sum_{k \geqslant 3}(k-4) P_{k} A_{j}(k, n)=K_{j+1}(n)+K_{j-1}(n)+P_{3}\left(A_{j+1}^{\text {down }}(3, n)+A_{j}^{\text {up }}(3, n)-A_{j-1}(3, n)\right)
$$


and, after subtracting twice (17), as:

$$
\begin{aligned}
\sum_{k}(k-6) P_{k} A_{j}(k, n)=K_{j+1}(n)-2 K_{j}(n)+K_{j-1}(n) \\
+P_{3}\left(A_{j+1}^{\text {down }}(3, n)+A_{j}^{\text {up }}(3, n)-A_{j-1}(3, n)\right) .
\end{aligned}
$$

Denoting by $V_{j}^{\text {down }}(n), V_{j}^{\text {up }}(n)$, the numbers of inward-, respectively outward-pointing vertices on the closed line separating layers $(j-1)$ and $j$ (figure 5), we have:

$$
K_{j}(n)=V_{j}^{\text {up }}(n)-P_{3} A_{j}^{\text {down }}(3, n)=V_{j+1}^{\text {down }}(n)+P_{3} A_{j}(3, n) .
$$

Thus,

$\sum_{k}(k-6) P_{k} A_{j}(k, n)=\left(V_{j+1}^{\mathrm{up}}(n)-V_{j+1}^{\text {down }}(n)\right)-\left(V_{j}^{\text {up }}(n)-V_{j}^{\text {down }}(n)\right)$

independently of the presence of three-sided cells.

Now, if one sums over all layers $1 \leqslant j \leqslant s$ and adds the contribution $(n-6)$ of the germ cell $j=0$ on the left-hand side, and $V_{1}^{\text {up }}(n)-V_{1}^{\text {down }}(n)=n$ on the right-hand side, one obtains:

$-Q_{s}(n)=(n-6)+\sum_{k}(k-6) P_{k} \sum_{j=1}^{s} A_{j}(k, n)=V_{s+1}^{\text {up }}(n)-V_{s+1}^{\text {down }}(n)-6$.

Each $k$-neighboured cell carries a topological charge $(6-k)$. The left-hand side is the total topological charge $Q_{s}=\sum_{\text {cluster }} N(k)(6-k)$ of the cluster of cells bounded by the closed contour separating layers $s$ and $(s+1)$.

$$
Q_{s}=6+V_{s+1}^{\text {down }}-V_{s+1}^{\text {up }} \text {. }
$$

The right-hand side is the outward flux of edges across the boundary. Equation (30) is therefore the topological form of Gauss's theorem, already introduced in Rivier and Aste (1996). Gauss's theorem is completely general, it holds for any closed contour, whether the foam is SSI or not, whether three-sided cells are present or not and if they are, whether $A_{j}(k, n)$ is linear or not. The construction by concentric layers is not necessary.

\section{Discussion of the simulations}

To test our predictions, we performed simulations of cellular networks, much like the simulations of Peshkin et al (1991). A cellular network of $10^{4}$ six-sided cells, with periodic boundary conditions, was constructed. We then performed $10^{5}$ random $\mathrm{T} 1$ in order to obtain a disordered system with a stationary distribution $P_{n}$. The topological correlators are computed in this final system. Further T1 are made to test the stationarity of the distribution. Alternatively, the procedure can be repeated many times to sample it. The stationarity of the distribution implies that the evolution of the system is ergodic. The correlators given below are the mean values of several runs. The ergodicity of the system was tested by comparing the results of the correlators obtained with a system of $10^{6}$ cells and the averaged values of 100 systems of $10^{4}$ cells. The results were identical. Note that our cellular network is purely topological, consisting of a connectivity graph of cell edges and three-fold vertices. Only the graph is retained during the simulation; there are no geometric or physical attributes, for example no vertex positions, edge curvatures, cell areas, or internal pressures.

We emphasize that the results of the topological simulations are artificial foams. We did not forbid a priori two neighbouring cells to share more than one side (decoration of a side). The analytical results established for layers $j$ with $j>1$ are true for SSI foams 
including three-sided cells only. However, we have not been able to find a set of topological moves to generate SSI foams, even with three-sided inclusions.

Furthermore, when the smallest number of sides allowed for a cell is four, simulations show that decoration of a side is very rare. The smallest edge-decorated cell then has seven sides. Thus, simulations without three-cells are very nearly natural foams, and, in general, SSI foams with very few defect inclusions. When the minimal number of sides allowed is five, the foam produced is even closer to a natural foam, and to a SSI foam with even less defect inclusions. We took advantage of this fact to test our results on simulations with foams whose cells have a minimal number of sides of four and five.

All the simulations recorded here include $99.9 \%$ of the cells. The proportion of cells with more than $n_{\max }$ neighbours is under $10^{-3} . n_{\max }=22$ when three-cells are allowed, $n_{\max }=18$ when they are not, and $n_{\max }=15$ when both three- and four-cells are forbidden.

Simulated foams are not SSI foams. They contain local defects, namely the cells in layer $j$ which are not in contact with layer $j+1$. Three-sided cells are always defects. Accordingly, the number of defects decreases for foams without three-cells, and even more for foams without three- and four-cells. In all cases, the number of defects in layer $j$ is found to vary linearly with $n$, the number of neighbours of the germ cell. The percentage of defects (ratio of the number of defects to the number of cells in the layer $j$ ) rapidly reaches a limiting value as a function of $j$, the distance from the germ cell.

\subsection{Linearity of the $A_{l}(k, n)$}

The first result of maximum entropy inference is the linearity of the correlator $A_{1}(k, n)$ for artificial foams and natural foams, provided that exploration of configuration space is not restricted by a dynamical constraint (such as context-dependent flips). Linearity of further correlators depends on that of $A_{1}(k, n)$. When no three-cells are allowed, all $A_{1}(k, n)$ are perfectly linear as shown in figure 6 . The simulations and theoretical predictions of maximum entropy are in perfect agreement in this case. When three-sided cells are allowed, the linearity of the $A_{1}(k, n)$ is only very good (it is excellent for small values of $k$ and only fair for high values) as shown in figure 7. (Figure 7 has the same data points as figure 4, but the dotted lines are here linear regressions.)

When neither three- nor four-sided cells are allowed, the foam is even closer to a natural foam (figure 8). The smallest edge-decorated cell has eight sides. Linearity of the $A_{1}(k, n)$ (figure 8) is perfect.

In all cases, Aboav's relation is entirely linear (figure 9).

\subsection{Comments on the linearity of the $A_{2}(k, n)$}

In figure 10 we plot the correlators $A_{2}(k, n)$. As predicted by the theory, they are linear in $k$ and in $n$. Figures 11 and 12 plot $A_{2}^{\text {arm }}(k, n)$ for the arm neighbours and $A_{2}^{\text {free }}(k, n)$ for the free neighbours. As expected, these two functions are bilinear in $k$ and $n$.

For comparison, figure 13 shows the mean number of second neighbours $\left(K_{2}(n)\right)$, of arm second neighbour $\left(K_{2}^{\text {arm }}(n)\right)$ and of free second neighbours $\left(K_{2}^{\text {free }}(n)\right)$ of a $n$-germ cell as a function of $n$, together with the mean number of first neighbours $\left(K_{1}(n)=n\right)$, for a foam free of three-cells. For a hexagonal tiling, $K_{1}(n)=K_{2}^{\text {free }}(n)=K_{2}^{\text {arm }}(n)=n=6$. $K_{2}^{\operatorname{arm}}(n)$ is always less than $K_{1}(n)$, and it has a smaller slope: even though an $n$ cell has $n$ arms, arm second neighbours may be connected with several arms to the germ cell (thereby decreasing the effective number of second arm neighbours). Indeed $K_{2}^{\text {arm }}=K_{2}-K_{2}^{\text {free }}=n-P_{4} A_{1}(4, n)<n$, for a foam free of three-cells. 


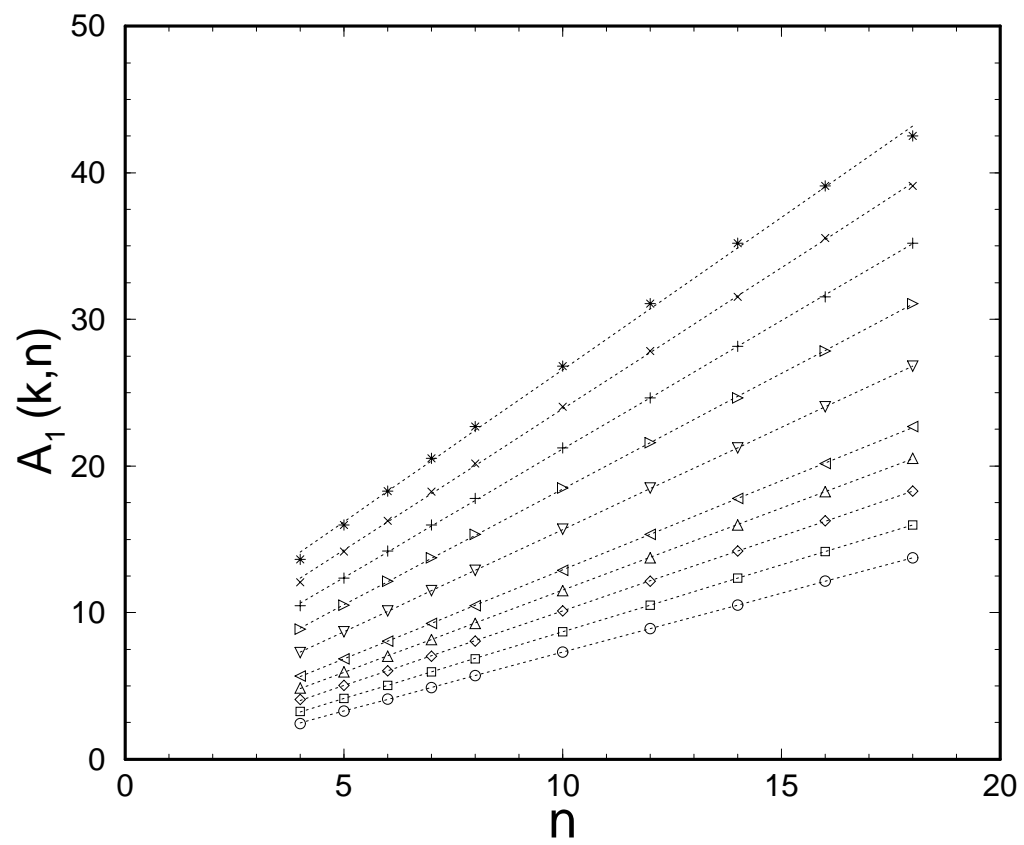

Figure 6. Nearest-neighbour correlators $A_{1}(k, n)$ for a foam without three-cells as a function of $n$ for $k=4$ (bottom line) to $k=18$ (top line). The dotted lines are the linear regression of each set of correlator. For $k>8$, only the $A_{1}(k, n)$ for $k$ even are plotted.

We can also note that there are always more free second neighbours than arm second neighbours, for any $n$. Thus, $K_{2}^{\text {free }}-K_{2}^{\text {arm }}=\sum_{k \geqslant 4}(k-6) P_{k} A_{1}(k, n)+2 P_{4} A_{1}(4, n)=$ $n(m(n)-6)+2 P_{4} A_{1}(4, n)>0$. Indeed, disorder brings in, on average, more seven-sided than five-sided nearest neighbours, i.e. an effective negative curvature for the first shell.

Figure 14 shows $A_{2}(k, n)$ in the general case of a system with three-sided cells and free of any known artificial constraints. $A_{2}(3, n)$ is perfectly linear in $n$, which confirms the hypothesis made in the previous section. For $k>3, A_{2}(k, n)$ depends linearly on $n$ as expected.

\subsection{Longer-ranged correlators}

The theory predicts a linear behaviour for the $A_{j}(k, n)$ (for any $j \geqslant 1$ ) for SSI foams (which are, by definition natural foams without defects). As pointed out at the beginning of this section, simulations only deal with artificial foams, but (cf figure 14) artificial foams without three-cells are very good approximations of natural foams without threecells. This is because the edge self-energies (two neighbouring cells sharing more than one edge) are much rarer; the decoration involves many more cells when three-sided cells are excluded. The linearity remains very good up to the sixth layer. (See, typically, the $A_{4}(k, n)$ in figure 15.) Further away, systematic deviations from linearity, associated chiefly, we believe, with artificial foams, become apparent as shown in figure 16.

Figure 15 confirms that artificial foams without three-cells are very good approximations of natural foams. Simulations of the former are in full agreement with the theoretical predictions made for the latter (SSI foams with defects). 


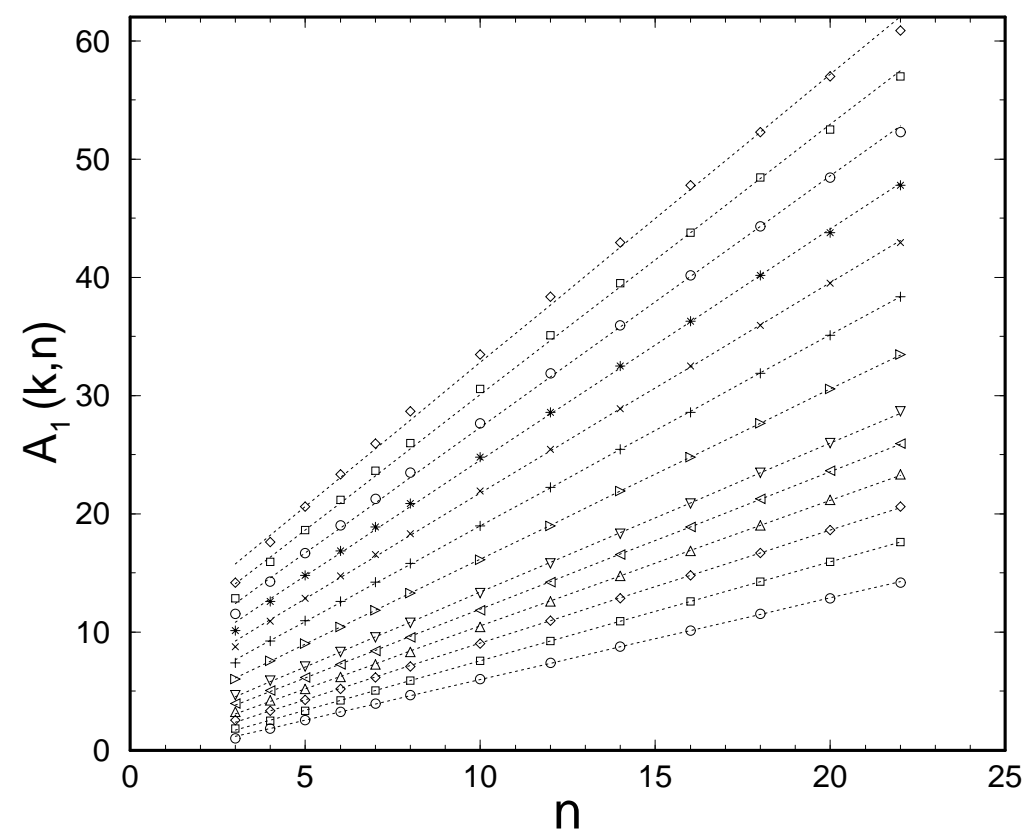

Figure 7. Topological correlators $A_{1}(k, n)$ for a foam without restriction as a function of $n$ for $k=3$ (bottom line) to $k=22$ (top line). The dotted lines are the linear regression of each set of correlators. For $k>8$, only the $A_{1}(k, n)$ for $k$ even are plotted.

\section{Conclusions}

The main result of this paper is a linear topological correlation between two cells in a foam in statistical equilibrium. Explicitly, the average number of $k$-neighboured cells at a distance of $j$ layers from a $n$-neighboured germ cell is linear in $n$. This linear correlation, which is a consequence of statistical equilibrium of the foam and its diagnostic, is obtained analytically by maximum entropy inference in the case of SSI foams, and verified in simulations for foams without any restrictions. It is a generalization of the correlation law obtained by Peshkin $e$ al for the $k$-neighboured nearest neighbours of a $n$-cell, which also implied the Aboav relation.

One distinguishes three classes of foams, artificial, natural and SSI. All natural foams are artificial foams, artificial foams which are not natural contain decorations of an edge (edge self-energies). Similarly, all SSI foams are natural foams. The natural foams which are not SSI contain local defects (among which three-sided cells are the most conspicuous). The analytic results have been obtained for SSI foams. We have studied the influence of three-sided defects, and shown that they do not affect the results. Simulations have been made on artificial foams. We have shown that artificial foams, without three-cells and with the local random variable $n$ counting the number of neighbours instead of the number of its sides, have the same statistical properties and correlations as natural foams. Notably, maximum entropy is applicable to either foams to infer the linearity of the twocells correlators $A_{1}(k, n)$, and of the Aboav relation, in a topological foam in statistical equilibrium under no other constraints than that, unavoidable, of filling space at random. Maximum entropy also infers the linearity of the two-cells correlators $A_{j}(k, n)$ for all $j>1$, but for SSI foams, including three-sided cells defects. All these linearities are confirmed 


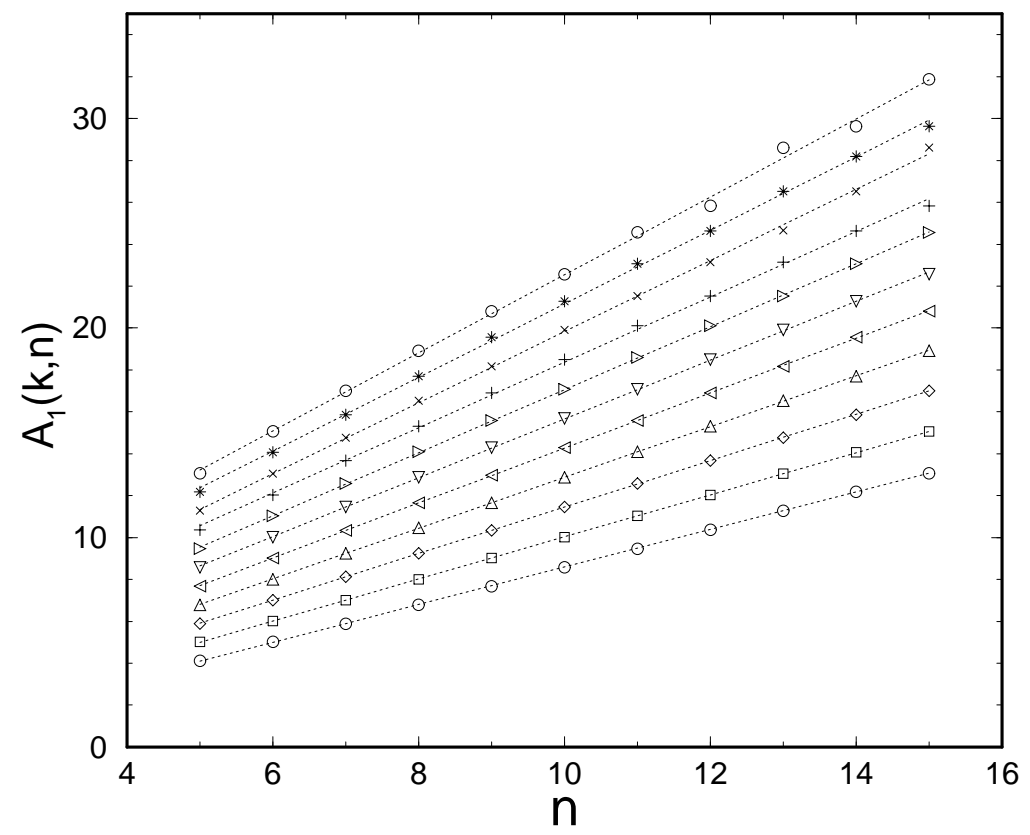

Figure 8. Topological correlators $A_{1}(k, n)$ for a foam without three- and four-cells as a function of $n$ for $k=5$ (bottom line) to $k=15$ (top line).

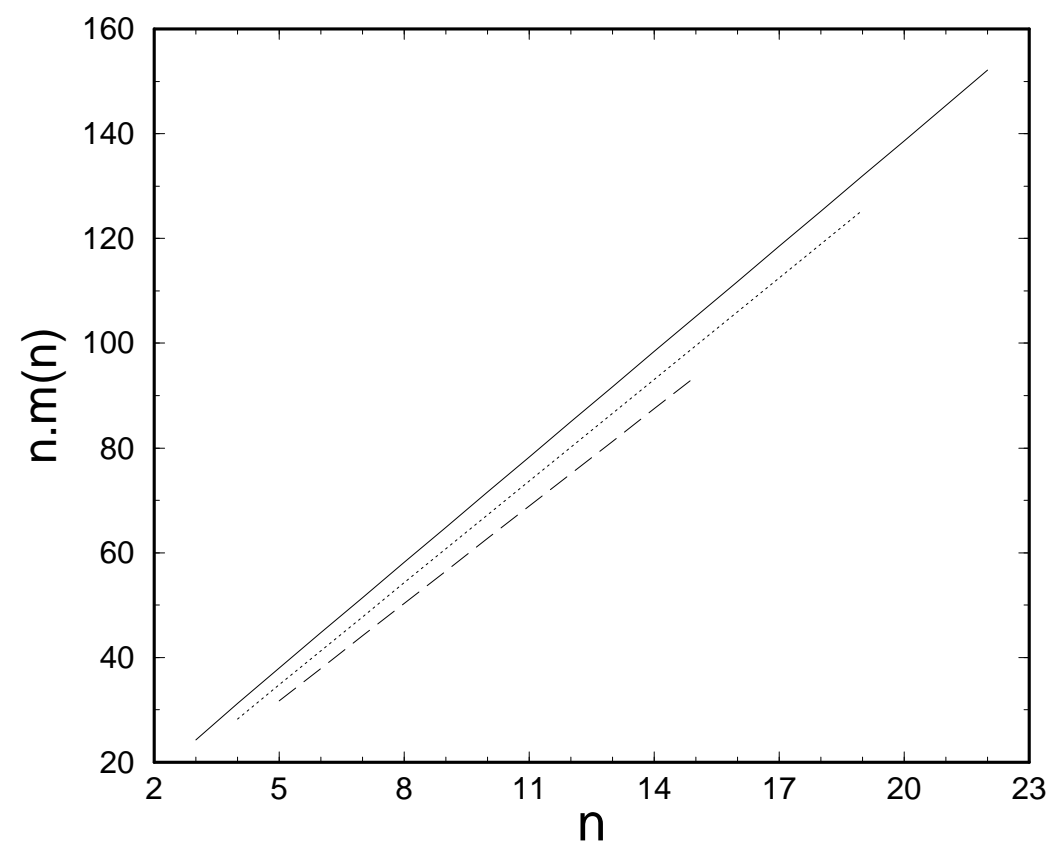

Figure 9. Aboav's relation for a foam without restriction (full line), without three-cells (dotted line) and without three- and four-cells (broken line). 


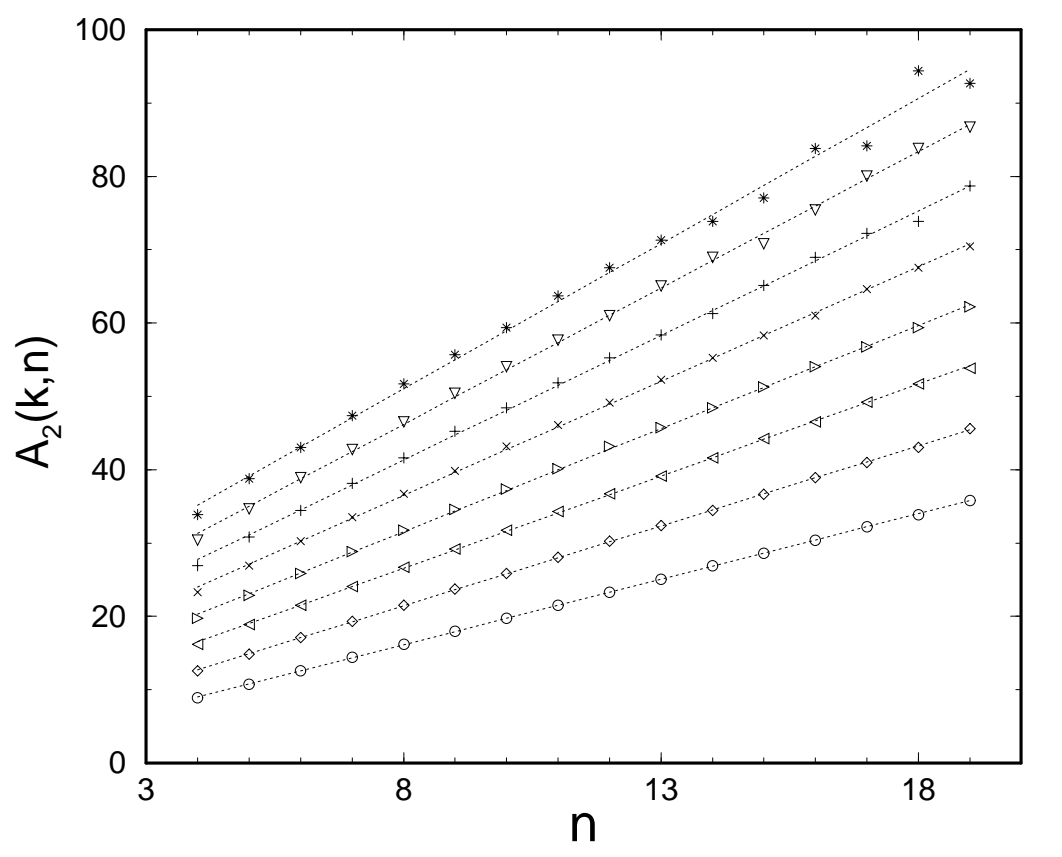

Figure 10. Second-neighbour correlators $A_{2}(k, n)$ (plotted for even $k$ only) for a foam without three-cells.

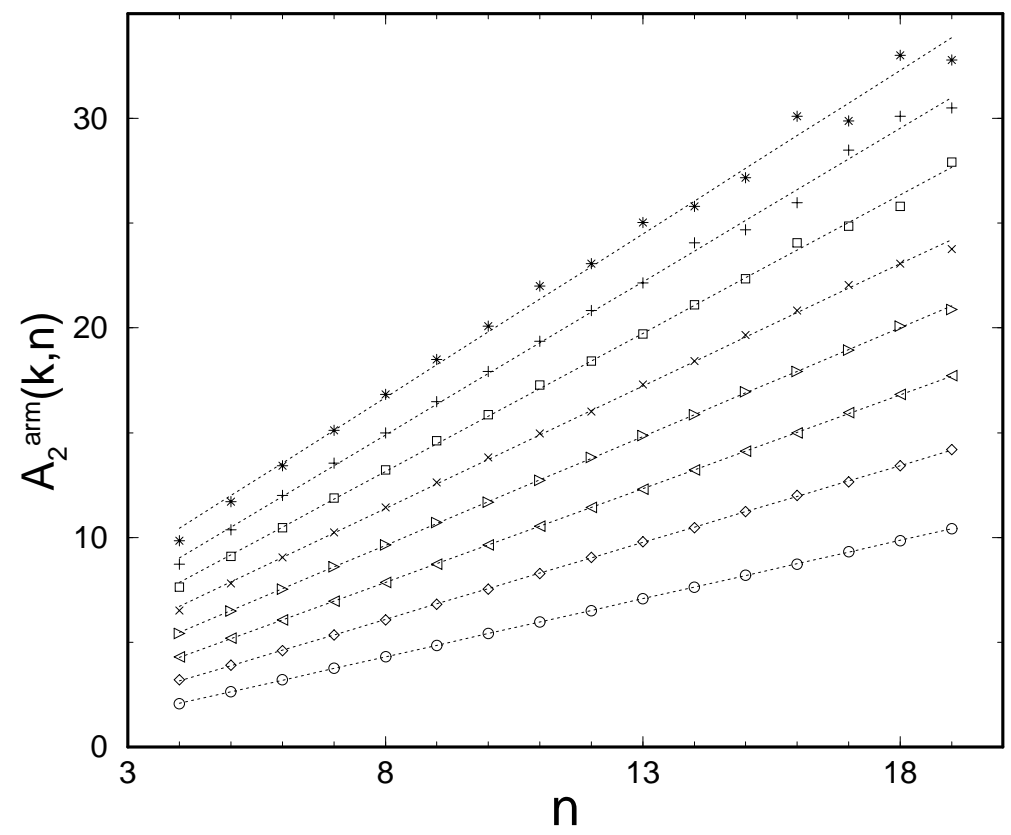

Figure 11. Arm second-neighbour correlators $A_{2}^{\text {arm }}(k, n)$ (plotted for $k$ even only) for a foam without three-cells. 


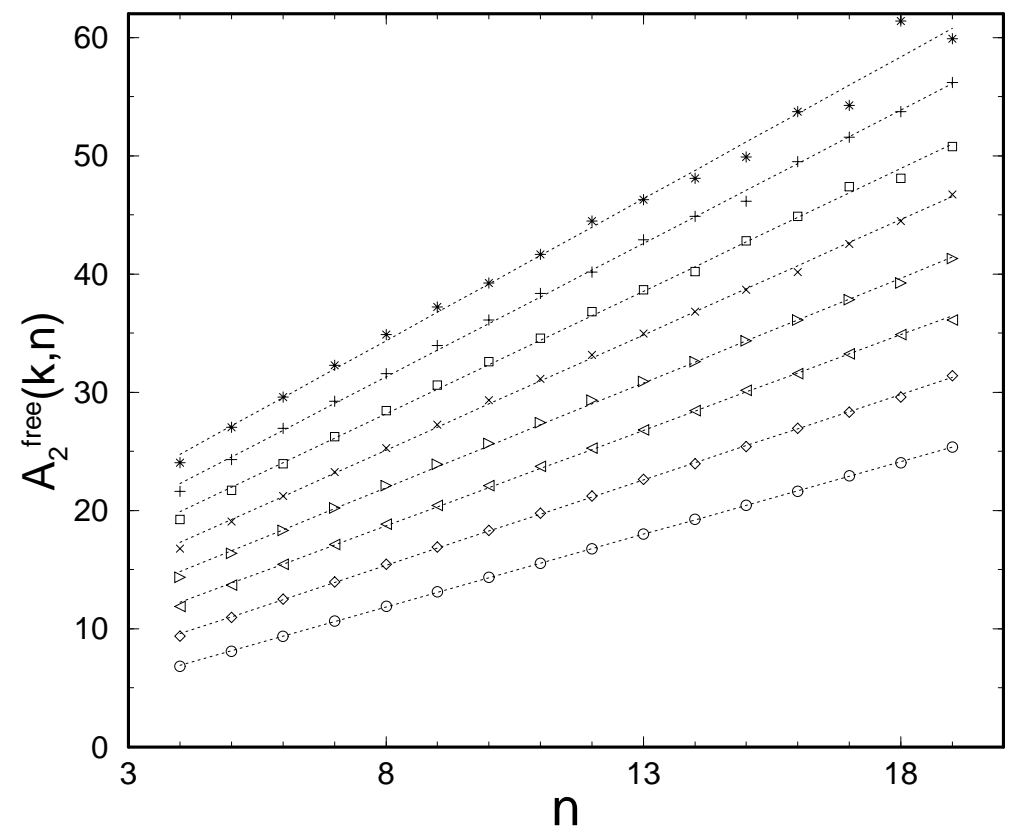

Figure 12. Free second-neighbour correlators $A_{2}^{\text {free }}(k, n)$ (plotted for $k$ even only) as a function of $n$ for $k=4$ (bottom line) to $k=18$ (top line) for a foam without three-cells.

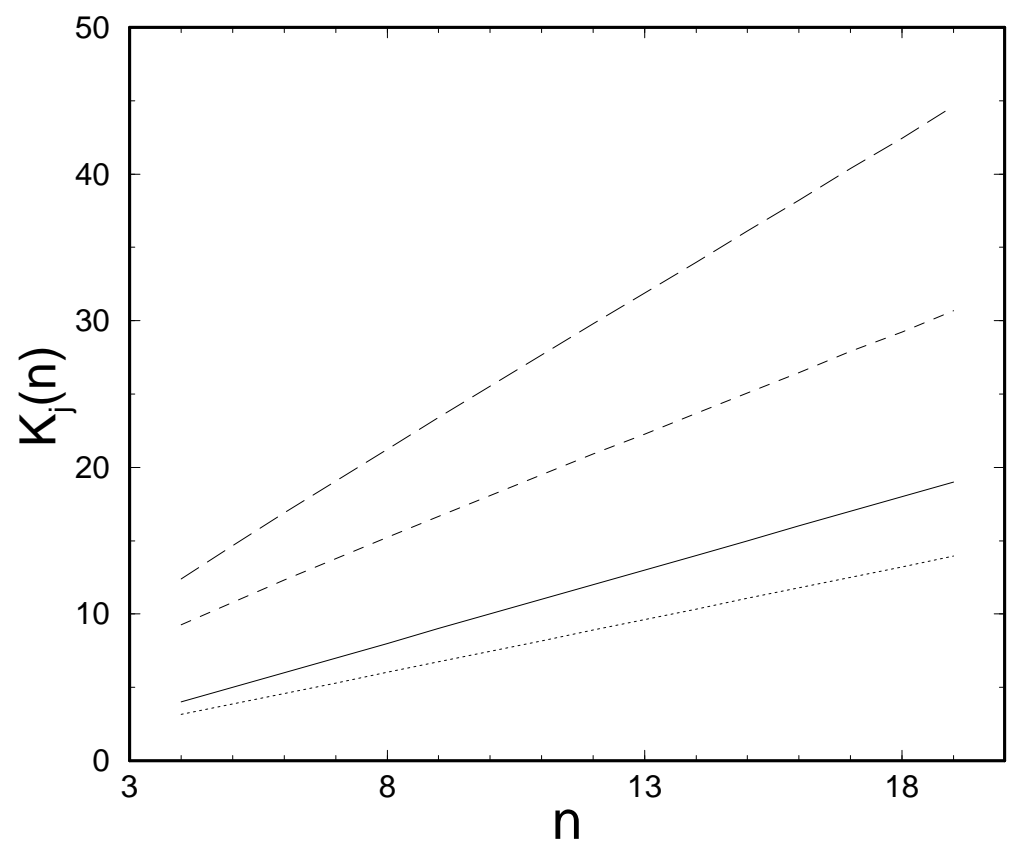

Figure 13. Average number of first neighbours (full line), of arm second neighbours (dotted line), of free second neighbours (broken line) and of second neighbours (long-broken line) of an $n$-cell, for a foam free of three-cells. 


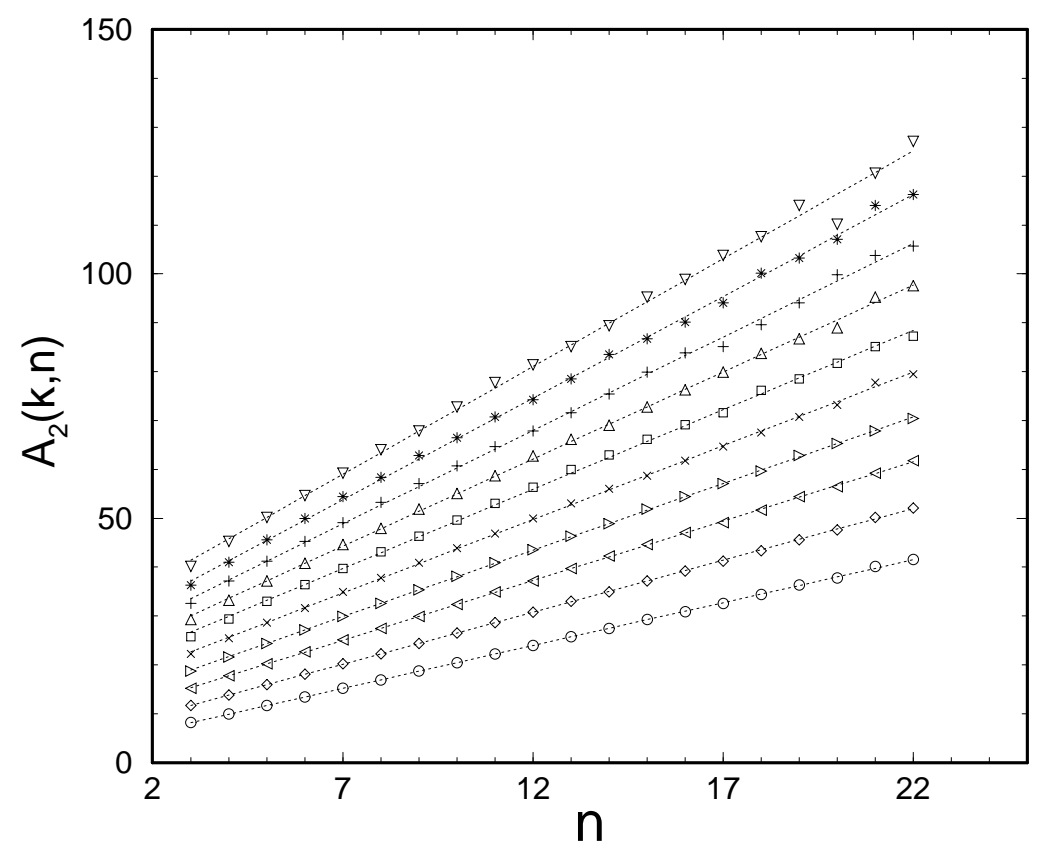

Figure 14. Second-neighbour correlators $A_{2}(k, n)$ (plotted for $k$ even only) for a foam without restriction.

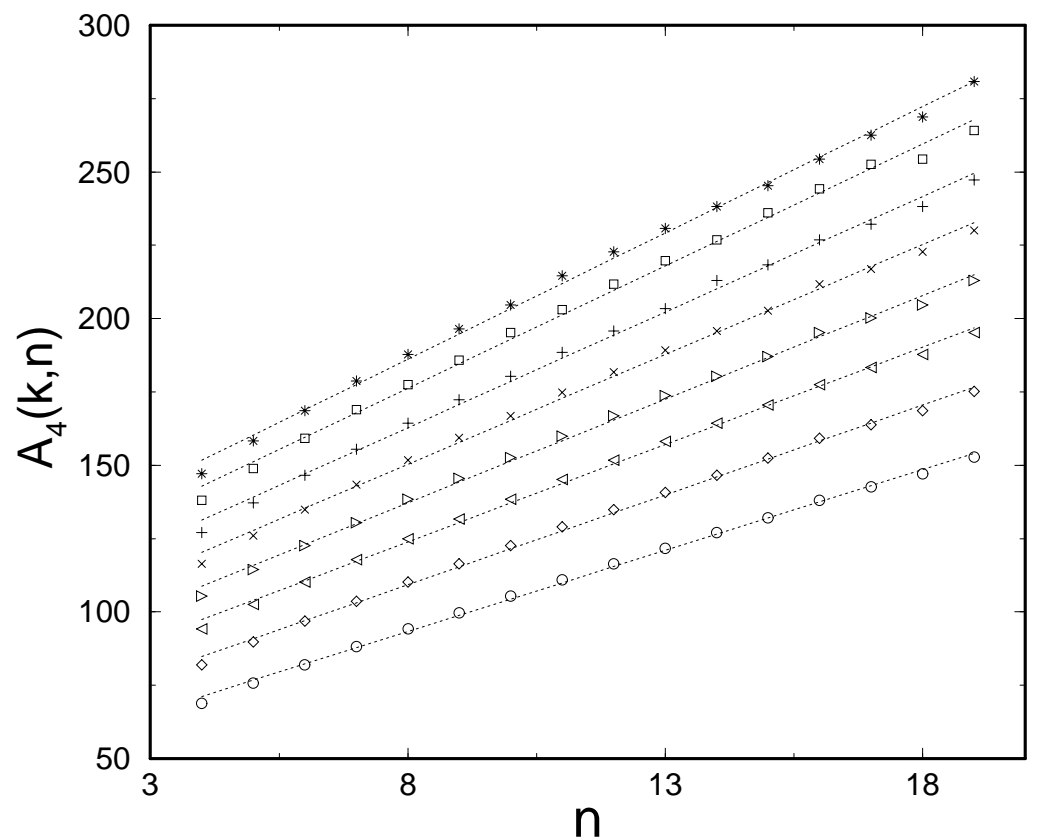

Figure 15. Topological correlators $A_{4}(k, n)$ for cells four layers apart (plotted for $k$ even only) for a foam without three-cells. 


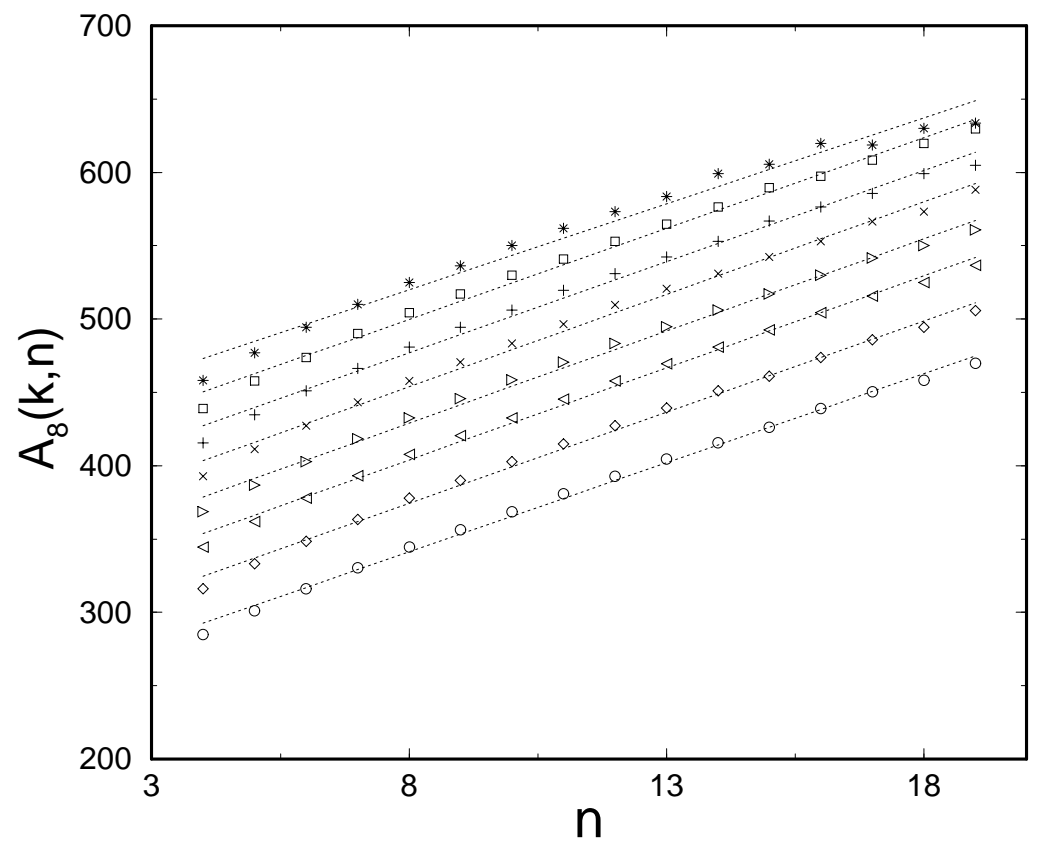

Figure 16. Topological correlators $A_{8}(k, n)$ for cells eight layers apart (plotted for $k$ even only) for a foam without three-cells. Simulations deal with artificial foams.

by the simulations (random topological edge-flips).

The simulations are very sensitive to any additional restriction in exploring local cell configurations, besides the unavoidable averaged topological constraints. Notably, we have shown that the slight deviation from linearity of Aboav's relation, observed by Godrèche et al is due to additional restrictions on topological edge-flips (in order to have a natural foam). When these additional restrictions are suppressed (we deal with an artificial foam), Aboav's relation is found to be strictly linear.

If deviations from the linearity of the correlators $A_{j}(k, n)$ are observed in a natural system, they are diagnostic of additional local restrictions (besides filling space at random) in the evolution of the system.

\section{Acknowledgments}

We are grateful to $\mathrm{T}$ Aste and R Delannay for many discussions and helpful comments.

\section{Appendix}

Maximum entropy inference is a well-defined and well-tested procedure (Jaynes 1957). One looks for the most probable distribution $p_{n}$ of the local random variable (here, the number of sides $n$ of a cell called the microscopic state, in a gas the velocity $v_{n}$ of an atom), subject to some information on the system. This information can be either of two types.

(i) Constraints on the probability distribution $p_{n}$. For example, in froths, $\sum_{n} p_{n}=1$ and $\sum_{n} n p_{n}=6$. In a gas, $\sum_{n} m v_{n}^{2}=U / N$, where $U / N$ is the average energy per particle. The left-hand side of the constraint is the average of a local 'microscopic' variable, and the 
right-hand side is the macroscopic, thermodynamic quantity which is kept constant under experimental conditions.

(ii) Prior knowledge on the system. This is most directly expressed in terms of an $a$ priori probability distribution $q_{n}$.

In a froth, no two- (or less-)sided cells are allowed, so that $q_{n \leqslant 2}=0$. In statistical thermodynamics, $q_{n}$ is the (normalized) multiplicity of state $n$. If there is a continuum of states, as with the velocity of an atom, $q\left(\epsilon=m v^{2} / 2\right)$ is called the density of states. One of the fundamental postulates of statistical thermodynamics is that all states $(\boldsymbol{x}, \boldsymbol{v})$ in a gas are equally probable a priori, i.e. that $q(\boldsymbol{x}, \boldsymbol{v})$ is a flat distribution. The state of a cell $(n)$ or an atom $(v)$ is shuffled by moves (collisions), which is a local process involving few cells or atoms. When the number of moves is sufficient so that all possible states have been explored, the system is in statistical equilibrium and its states will be distributed according to $p_{n}$, which maximizes the entropy

$$
S=-\sum_{n} p_{n} \ln \left(\frac{p_{n}}{q_{n}}\right)
$$

subject to constraints (i). The constraints $\left(\sum_{n} n p_{n}=6\right.$, say) are ensured by a Lagrangian multiplier ( $\beta$, say), and the equilibrium distribution is given by:

$$
p_{n}=\frac{q_{n} \exp \left(-\beta_{n}\right)}{\sum_{n} q_{n} \exp \left(-\beta_{n}\right)} .
$$

It is moulded both by constraints (i) and by the prior knowledge $q_{n}$ (ii). In addition, maximum entropy inference yields equations of states, i.e. relations between the various constraints (for example, Aboav's law, or $P=\frac{2}{3}(U / V)$ in a gas of volume $V$, where both the energy $U$ and pressure $P$ are averages of $v^{2}$ ).

Prior knowledge can also be given in terms of transition probabilities for a move, i.e. by the kernel $\Gamma\left(n \rightarrow n^{\prime}\right)$ relating the initial state $n$ and the final state $n^{\prime}$ of the cells involved in the move, or of the atoms involved in the collision. For example, in a Monte Carlo simulation, a move is made with probability 1 if the energy of the final state is lower than that of the initial state, but only with probability $\exp (-\beta \Delta \epsilon)$, if the energy of the final state is higher by $\Delta \epsilon$. In froths, when the move is the division of a cell, prior knowledge is the probability that an $n$-sided cell divides, and the kernel of division of an $n$-sided cell into an $n^{\prime}$-sided daughter (the other having $n+4-n^{\prime}$ sides). This prior knowledge has a considerable effect on the equilibrium cell distribution (Dubertret et al 1997). It could, of course, be expressed, but a posteriori (from equation (A2)), in terms of a prior probability $q_{n}$. That is not very illuminating. We stress that prior knowledge involves a priori probability or multiplicity $q_{n}$, and also a transition kernel which is the same for all moves, and depends only on the local states of a cell, atom, etc before and after the move. It is therefore strictly a transition probability in a Markovian process.

In our simulations, prior knowledge consists of $q_{2}=0$. (Two-sided cells are forbidden, and thus any move leading to a two-sided cell is also forbidden.) Apart from that, all moves (T1, or neighbour switch) are equiprobable (of course, a cell with many sides has more chances of being affected by a move, because edges are selected at random). The resulting distribution (which we do not calculate here) would be affected by different transition probabilities (as shown in Dubertret et al (1997)), but neither the fact that the system reaches statistical equilibrium, nor its equation of state.

Now, where do the simulations of Godrèche et al stand with respect to maximum entropy? Godrèche et al rejected the moves (flip) which would lead to a tadpole or the renormalization of an edge (self-energy inclusion), while we accept these moves (except if 
a two-sided cell is involved in the tadpole or the renormalized edge). Renormalization of an edge implies that two cells are neighbours through more than one edge; tadpole, that one cell is its own neighbour. The move (flip) leading to a final state involving edge renormalization is forbidden, but this final state is not characterized by the local random variable $n^{\prime}$. It is qualified by a context-dependent, non-local specification. This move is non-Markovian, it is not a transition probability between an initial and final state, and the rejection criterion is not prior knowledge. Moreover, a tadpole can only be obtained by flipping a self-energy inclusion. Consequently, the simulations of Godrèche et al are not exploring the possible states of the cells in a context-free fashion, dependent only on the local state of the system. Maximum entropy inference cannot be applied to a froth generated in this way.

\section{References}

Aboav D A 1970 The arrangement of grains in a polycrystal Metallogr. 3383

Aste T, Boose D and Rivier N 1996a From one bubble to the whole froth: A dynamical map Phys. Rev. E 53 6181-91

Aste T, Szeto K and Tam W 1996b Statistical properties and shell analysis in random cellular structures Phys. Rev. E 54 5482-92

Delannay R and Le Caër G 1994 Topological characteristics of 2d cellular structures generated by fragmentation Phys. Rev. Lett. 73 1553-6

Dubertret B, Aste T, Ohlenbusch H and Rivier N 1997 Two-dimensional froths and the dynamics of biological tissues Phys. Rev. E submitted

Dubertret B and Rivier N 1997 The renewal of the epidermis: A topological mechanism Biophys. J. 73 38-44

Gibson L J and Ashby M 1988 Cellular Solids (New York: Pergamon)

Godrèche C, Kostov I and Yekutieli I 1992 Topological correlations in cellular structures and planar graph theory Phys. Rev. Lett. 69 2674-7

Jaynes E 1957 Information theory and statistical mechanics, i Phys. Rev. 106 620-30

Jaynes E 1957 Information theory and statistical mechanics, ii Phys. Rev. 108 171-90

Le Caër G and Delannay R 1993 Correlations in topological models of 2d random cellular structures J. Phys. A: Math. Gen. 26 3931-54

Lewis F 1928 The correlation between cell division and the shapes and sizes of prismatic cells in the epidermis of Cucumis Anat. Rec. 38 341-76

Mullins W 1956 J. Appl. Phys. 27900

Ohlenbusch H, Aste T, Dubertret B and Rivier N 1997 Topological structures of disordered cellular systems Eur. J. Phys. B submitted

Peshkin M A, Strandburg K J and Rivier N 1991 Entropic prediction for cellular networks Phys. Rev. Lett. 67 1803-6

Rivier N 1993a Geometry and evolution of biological tissues Continuum models of Discrete Systems vols 123-5, ed K-H Anthony and H-J Wagner (Trans Tech Publication) pp 383-92

Rivier N 1993b Order and disorder in packings and froths Disorder and Granular Media ed D Bideau and A Hansen (Amsterdam: North-Holland) pp 55-102

Rivier N and Aste T 1996 Curvature and frustration in cellular systems Phil. Trans. R. Soc. A 354 2055-69

Rivier N, Dubertret B, Aste T and Ohlenbusch H 1998 in preparation

Rivier N and Lissowski A 1982 On the correlation between sizes and shapes of cells in epithelial mosaics J. Phys. A: Math. Gen. 15143

Stavans J and Glazier J 1989 Phys. Rev. Lett. 621318

Szeto K, Aste T and Tam W 1997 Topological correlations in soap froths Phys. Rev. submitted

Thompson d'A W 1942 On Growth and Form 2nd edn (New York: Cambridge University Press)

Tutte W 1962 A census of planar triangulations Can. J. Math. 1421

Weaire D 1974 Metallography 7157

Weaire D and Rivier N 1984 Soap cells and statistics-Random patterns in two dimensions Contemp. Phys. 25 59-99 